\title{
Digital technologies and adherence in respiratory diseases: the road ahead
}

\author{
John D. Blakey ${ }^{1}$, Bruce G. Bender ${ }^{2}$, Alexandra L. Dima³ ${ }^{3}$ John Weinman ${ }^{4}$, \\ Guilherme Safioti ${ }^{5}$ and Richard W. Costello ${ }^{6}$
}

Affiliations: ${ }^{1}$ Respiratory Medicine, Royal Liverpool Hospital and Health Services Research, University of Liverpool, Liverpool, UK. ${ }^{2}$ Dept of Pediatrics, National Jewish Health, Denver, CO, USA. ${ }^{3}$ Health Services and Performance Research, Université Claude Bernard Lyon 1, Lyon, France. ${ }^{4}$ Institute of Pharmaceutical Science and Institute of Psychiatry, Psychology and Neuroscience, King's College London, London, UK. ${ }^{5}$ Teva Pharmaceutical Industries Ltd, Amsterdam, The Netherlands. ${ }^{6}$ RCSI Education and Research Centre, Royal College of Surgeons in Ireland, Beaumont Hospital, Dublin, Ireland.

Correspondence: Richard W. Costello, RCSI Medicine, RCSI Education and Research Centre, Royal College of Surgeons in Ireland, Beaumont Hospital, Beaumont, Dublin 9, Ireland. E-mail: rcostellodrcsi.ie

@ERSpublications

Digital technologies hold promise to improve adherence and personalise care in patients with respiratory diseases http://ow.ly/WjTz30m71ZW

Cite this article as: Blakey JD, Bender BG, Dima AL, et al. Digital technologies and adherence in respiratory diseases: the road ahead. Eur Respir J 2018; 52: 1801147 [https://doi.org/10.1183/ 13993003.01147-2018].

ABSTRACT Outcomes for patients with chronic respiratory diseases remain poor despite the development of novel therapies. In part, this reflects the fact that adherence to therapy is low and clinicians lack accurate methods to assess this issue. Digital technologies hold promise to overcome these barriers to care. For example, algorithmic analysis of large amounts of information collected on health status and treatment use, along with other disease relevant information such as environmental data, can be used to help guide personalised interventions that may have a positive health impact, such as establishing habitual and correct inhaler use. Novel approaches to data analysis also offer the possibility of statistical algorithms that are better able to predict exacerbations, thereby creating opportunities for preventive interventions that may adapt therapy as disease activity changes. To realise these possibilities, digital approaches to disease management should be supported by strong evidence, have a solid infrastructure, be designed collaboratively as clinically effective and cost-effective systems, and reflect the needs of patients and healthcare providers. Regulatory standards for digital interventions and strategies to handle the large amounts of data generated are also needed. This review highlights the opportunities provided by digital technologies for managing patients with respiratory diseases. 


\section{Current and emerging digital technologies in respiratory medicine}

In the context of respiratory disease, poor adherence to medication is a significant concern associated with poor patient outcomes, unnecessary escalation of medication and increased healthcare costs [1]. Complex treatment regimens for patients with asthma and COPD, including both "as needed" medications for acute symptoms and long-term maintenance medications in a mixture of device types, are among the barriers to adherence [2-4]. Furthermore, optimal self-management often requires multiple components in addition to adherence, such as measuring lung function and recording symptoms, which are not easy for patients to maintain. To address the need for optimised adherence to maintenance medication in patients with respiratory diseases, a number of digital technologies have been developed. Strategies used to date include approaches to monitoring and improving adherence, such as electronic inhalers, text messaging and reminders, and self-management tools (such as web-based and mobile applications to record symptoms and monitor lung function) [5-8]. Examples of digital interventions targeting adherence in patients with asthma and COPD that were published between 2007 and 2017, including electronic monitoring devices (EMDs), text messaging, and web and mobile applications, are summarised in table 1. However, technology is rapidly advancing and additional features and enhancements are continually being developed.

These advancements include strategies to better monitor adherence. In patients with CF, chipped-nebulisers are available that provide objective date- and time-stamped adherence data [36]. Inhalers and inhaler add-ons (digital devices attached to an existing inhaler) designed to measure and assess inhaler technique/quality of inhalation are also available, which can help identify and overcome unintentional poor adherence $[1,37,38]$. Smart inhalers have also been developed that wirelessly send data on inhaler usage directly to a mobile health platform or website $[1,3]$. Such platforms offer several advantages, including visualisation of measurements and integration into a wider dataset, such as that contained in a patient's electronic medical records [25, 39].

Technologies are also available that remotely monitor physiological parameters, including Bluetooth-connected devices and mobile applications that measure peak flow, exhaled nitric oxide fraction $(F$ eNO), physical activity and ambient pollution. These data can link adherence management with other aspects of patient self-management $[40,41]$ and may be used to provide appropriate information to promote healthy behaviours (such as warning individuals of changes in ambient pollution that may require them to use their preventer treatment and carry their reliever treatment). Such connected information may also provide mechanistic insights into the effect of treatment adherence on health outcomes [42]. New and detailed information can also be obtained from wearable biosensors that continuously monitor respiratory and cardiac parameters using acoustic signals [43]. Taken together, digital approaches targeting adherence and advances in physiological monitoring of disease open up a range of possibilities for understanding the causes and consequences of poor adherence and, hence, a rational way to deliver effective adherence management.

\section{Potential opportunities provided by digital technologies in respiratory medicine}

Several attributes of digital technologies, which could be summarised under the headings of precision, penetration, prediction and personalisation, suggest how current and future technologies may be incorporated into the multidimensional nature of healthcare.

\section{Precision}

Longitudinal data on patients' adherence collected via digital applications, particularly when supported by digitally collected information on symptoms and physiological and environmental parameters, can provide a detailed and precise basis for understanding an individual's disease [44, 45]. For example, in patients with asthma, digitally collected real-time data on adherence and symptoms can help HCPs differentiate between symptoms or changes in lung function due to low adherence versus those indicating refractory disease or disease progression [46]. This is illustrated in figure 1, which shows patterns of change in lung function based on digitally collected data on inhaler technique and inhaler use coinciding with peak expiratory flow (PEF). Based on such data, HCPs can identify patients for whom additional training on inhaler technique or interventions to optimise inhaler use may be beneficial. Additionally, identifying inconsistencies between recorded data on symptom severity and measures of lung function may lead HCPs to assess and adjust both reliever and controller medication. Such data can help HCPs to differentiate between low implementation (short periods with no medication use) and nonpersistence (medication discontinuation) followed by re-initiation. Digital solutions that effectively link symptoms, data recorded by electronic peak flow meters (as a substitute for measures of lung function) and adherence (measured by digital records of medication use) can be helpful in terms of accurately assessing medication use, as well as in helping patients understand and self-manage their disease. Such an approach is analogous to how a patient with diabetes alters insulin therapy in response to glucose levels based on day-to-day 


\section{Electronic monitoring devices (EMDs)}

CHARLES et al. 2007 [6]

24-week RCT

110 patients laged 12-

65 years) with asthma

BuRgess et al. 2010 [9]

4-month RCT

26 patients laged 6-14 years) with suboptimal asthma control

FosTer et al. 2014 [10] 6-month RCT

143 patients laged 1465 years) with suboptimal asthma control

CHAN et al. 2015 [5]

6-month RCT

220 children laged 6-15 years) with prior history of asthma exacerbation

VASBINDER et al. 2016 [11]

12-month RCT

209 children laged 4-11 years) with asthma

MERChANT et al. 2016 [12]

12-month parallel arm study

495 patients (aged $>5$ years) with asthma

MORTOn et al. 2017 [13]

1 -year RCT

90 children (aged $6-16$ years) with asthma
EMD recorded date/time and number of actuations Intervention group also utilised an audiovisual reminder function

EMD recorded date and time of actuation Intervention group also received feedback based on EMD measured adherence

EMD recorded date/time of all actuations and uploaded data monthly to a secure website Intervention group also received IRF and/or personalised adherence discussions

EMD recorded date/time and number of actuations Intervention group also utilised an audiovisual reminder function

EMD recorded date and time of actuation Intervention group also received tailored SMS

reminders when a dose was at risk of omission

Intervention included a sensor to monitor inhale use, an education component, reminders and alerts, data visualisation and trends, and predictive analytics and feedback

Control group utilised sensors to monitor inhaler use along with usual care

EMD recorded date and time of actuation Adherence data also reviewed by patient and caregiver every 3 months and reminders utilised in the intervention group
Significantly higher adherence in patients who received an audiovisual reminder compared with the control group $193 \%$ versus $74 \%$ median percentage taken in last 12 weeks of trial, $p<0.001$ )

Significantly higher adherence in patients who received feedback compared with the control group $(79 \%$ versus $58 \%, p<0.01$ )

Adherence in the control group declined slightly over the study, whereas in the intervention group mean adherence was maintained $(p<0.01)$

Significantly higher adherence in the IRF groups than in the non-IRF groups $173 \%$ versus $46 \%$ of prescribed daily doses; $p<0.0001)$

Adherence decreased over time in all groups, but remained twice as high in the IRF groups versus the non-IRF groups $160 \%$ $\pm 38 \%$ versus $29 \% \pm 33 \%$ )

Significantly higher adherence in the intervention group compared with the control group (median $84 \%$ versus $30 \%$, $p<0.001$ )

Overall adherence fell in both groups over time, with no difference in the rate of decline $(p=0.10)$

Higher mean adherence in the intervention group compared with the control group $169.3 \%$ versus $57.3 \%$; difference $12.0 \%$, $95 \% \mathrm{Cl} 6.7 \%-17.7 \%$ )

Greater decrease in mean daily SABA uses per person in the intervention group compared with routine care $(-0.41$ versus $-0.31, p<0.0011$

Greater increase from baseline in the mean percentage of SABA-free days in the intervention group compared with routine care $(21 \%$ versus $17 \%, p<0.01)$

Significantly higher adherence in the intervention group compared with the control group $(70 \%$ versus $49 \%, p \leqslant 0.001]$

Adherence was maintained in the intervention group, but declined in the control group over 1 year
No significant differences occurred in clinical outcomes between the two groups

Change in forced FEV1 was greater in the intervention group (13.8\%) than in the control group (9.8\%), but did not reach statistical significance

$11 \%$ of patients in the IRF groups had exacerbations compared with $28 \%$ in the non-IRF groups ( $p=0.013)$. This difference was not significant ( $p=0.06)$ after adjustment for clustering and past self-reported prednisone use

Reduction in asthma morbidity score from baseline to 6 months was significantly greater $(p=0.008)$ in the intervention group (mean score of 9.3 at baseline versus 7.3 at 6 months) than in the control group (mean score of 9.2 at baseline versus 8.0 at 6 months)

No differences were found between groups for asthma control, QoL, or asthma exacerbations

Improved ACT scores among adults initially lacking asthma control

Significant decrease in exacerbations requiring oral steroids or hospitalisation in the intervention group

Decrease in ACQ in both groups (1.0 in the control group and 0.9 in the intervention groupl but no significant difference between groups 


\section{TABLE 1 Continued}

\section{Text messaging}

StRANDBYGAARD et al. 2010 [14]

12-week RCT

26 patients (aged $18-45$ years) with asthma

PRABhakaran et al. 2010 [15]

12-week RCT

120 patients laged $>21$ years hospitalised for asthma

Lv et al. 2012 [16]

12-week RCT

150 patients laged $>18$ years) with asthma

Petrie et al. 2012 [17]

9-month RCT

147 patients laged

16-45 years) with asthma

Kolmodin MAcDonell et al 2016 [18]

3-month RC

48 African American adults (aged 18-29 years) with asthma

\section{BRITTo et al. 2017 [19]}

6-month RCT

64 patients (aged 12-22 years) with asthma

Akrom et al. 2015 [20]

Controlled pre- and

post-intervention study

66 patients laged $18-80$ years) with COPD
Intervention group received daily SMS reminder to take asthma medication

All patients received inpatient individualised asthma education at the beginning of the study

Intervention group received SMS messages to

assist with asthma management, including medication use, according to a structured workflow

Verbal asthma education (all groups)

Individualised asthma action plan with PEF monitoring and asthma diary (traditional and SMS groups)

Daily SMS reminders on how to manage asthma and option to ask questions via text message (SMS group)

Treatment group received individually tailored text messages for 18 weeks based on their illness and medication beliefs

ntervention group received two computer-delivere motivational interviewing (MI) sessions with text reminders between sessions

Control group completed asthma education matched for length, location and method of delivery of the intervention group MI sessions

Intervention group received text message reminders personalised by the patient

Ability to change, add, or delete reminders as desired

Participants divided into intervention from baseline to month 3 or intervention from month 3 to month 6

Intervention group received daily text messages with motivational messages and reminders to take medication, and brief counselling Control group received hospital standard of care
Mean medication adherence increased in the SMS group $(77.9 \%$ to $81.5 \%)$ and decreased in the control group $(84.2 \%$ to $70.1 \%$ )

The absolute difference in mean medication

adherence between the two groups was

$17.8 \%(p=0.019)$

Not measured
Medication adherence was higher in the SMS $(80.0 \%)$ and traditional $(74.1 \%)$ groups than in the control group $(50.0 \%)$, but changes were not significant

Follow-up appointment rates were significantly higher in the SMS $(60 \%)$ and traditional $(54 \%)$ groups compared with the control group (28\%)

Average self-reported adherence was higher in the intervention group than the control group $(57.8 \%$ versus $43.2 \%, p<0.05)$

Percentage taking over $80 \%$ of prescribed inhaler doses was $25.9 \%$ in the intervention group and $10.6 \%$ in the control group $(p<0.05)$

Both groups missed fewer doses of controller medication at 3 months than at baseline

The magnitude of the trend was greater for the intervention group for total doses missed, average doses missed per day and number of days medication was missed, but did not reach statistical significance

Increased adherence to ICS by $2.75 \%$ per month with the intervention versus without it $(p<0.01)$

For the group that received text messages first, adherence subsequently declined, suggesting no long-term effect

Intervention group experienced significant improvement in MMAS score from pre-test to post-test ( $46 \%$ to $88 \%$ high compliance): however, the control group did not $(55 \%$ to $61 \%$ high compliance)
No between-group differences in change in $\mathrm{FeNO}_{\mathrm{N}}$ FEV1 (\% predicted), ACQ, or mini-AQLQ

No significant difference in ACT scores, number of nebulisations, or ED visits between groups

Significant increase in perceived asthma contro and $A Q L Q$ in the SMS and traditional groups relative to the control group

Improved FEV1 (\% predicted) in all groups, but no significant between-groups difference

At 18 weeks, the text message group was significantly higher than the control group on perceived necessity of preventer medication, belief in the long-term nature of their asthma and perceived control over their asthma

There was a larger magnitude decrease in symptoms in the intervention versus the control group, both in total symptoms ( $p<0.05)$ and average symptoms per day $(p<0.05)$

FEV 1 (\% predicted) improved in the intervention group and deteriorated in the control group $1+4.41 \%$ and $-4.14 \%$, respectively, $\mathrm{p} \leqslant 0.01]$

Improved ACT score after 1 month that was maintained for the 6-month duration of the study in both groups

Asthma symptoms improved and asthma worry decreased in both groups

Not measured 
Web-based and mobile applications

VAN DER MeER et al. 2009 [21]

12-month RCT

200 patients laged 18-

50 years) with asthma

Liv et al. 2011 [22]

6-month prospective,

controlled study

120 patients with asthma

Ryan et al. 2012 [23]

6-month RCT

288 patients (aged $>12$ years)

with asthma

FAROOQUI et al. 2015 [24]

30-day single-arm study

24 patients (aged 9-16 years)

with asthma; 21 patients

completed the study

Mosnaim et al. 2015 [25]

8-week single-arm study

12 African American patients

laged 11-16 years) with

persistent asthma

CINGI et al. 2015 [26]

3-month RCT

136 patients laged 25-41

years) with asthma
Internet-based self-management program included weekly asthma control monitoring, treatment advice, online and group education, and remote communication with an asthma nurse

Control group received the usual care

Mobile phone-based interactive asthma self-care system including electronic symptoms diary and record of reliever use and PEFR

Control group received a written symptoms bookle and were asked to record PEFR regularly

Mobile phone-based system with twice-daily recording and transmission of symptoms, drug use and peak flow, with immediate feedback

Control group recorded the same data using a paper diary

Intervention included:

Daily reminders for medication use

Personalised trigger avoidance measures

Algorithm-based, interactive asthma treatment plan

Gamification features and reward points based on

medication use and interaction with the app

Intervention included:

Daily visual reminders to take their ICS

Positive reinforcement (texts and gaming) for taking ICS

Immediate (ability to customise avatar) and long-term rewards $\$ \$ 1.00$ per dose to purchase music, movies, applications and games)

Intervention included physician/patient

communication, health status and medication

compliance tracking, sharing of motivational and

educational content, and medication reminders Control group received standard care
Inhalation technique improved in both groups but improvements did not differ between groups ( $p=0.143$ )

Self-reported medication adherence did not differ between groups

Significant increase in mean daily dose of either systemic corticosteroids or ICS in intervention group compared with control group

Not measured

Increased adherence to controller medication in 18 out of 21 patients $(85 \%)$ during the intervention period compared with the

30 days immediately preceding enrolment

Median ICS adherence increased from 19\% at baseline to $67 \%$ at 8 weeks

At baseline $8 \%$ of patients met target ICS adherence ( $>50 \%$ ), while at 8 weeks, $58 \%$ of patients met target ICS adherence

Not measured

(18 versus 23 ) with $58 \%$ of participants achieving the minimal clinically important difference (3 points) in ACT score

SABA use decreased from a median of 3 puffs per week at baseline to 0 puffs per week at 8 weeks

In the intervention group, more patients achieved a well-controlled asthma score (ACT >19) compared with the control group $149 \%$ versus $27 \%, p<0.05$ ) 


\section{TABLE 1 Continued}

JoHnson et al. 2016 [27]

3-week RCT

98 patients (aged 12-17 years) with asthma

AHmed et al. 2016 [28]

6-month RCT

100 patients laged 18-

69 years) with asthma

Cook et al. 2016 [29]

Prospective single-arm,

treatment-only, 4-month study

60 adults (aged 17-82 years) with asthma

Koufopoulos et al. 2016 [30] 9-week RCT

216 patients laged 18

64 years) with asthma

KIM et al. 2016 [31]

8-week study

44 patients (aged $>19$ years) with asthma

MoRRISOn et al. 2016 [32]

12-week RCT

51 patients laged $\geqslant 16$ years) with asthma

Pool et al. 2017 [33]

12-month RCT

408 adults (aged $21-60$ years)

with asthma
Personalised health application (MyMediHealth) to help patients manage medications and receive dosing reminders

Control group received action lists as part of usual care

My Asthma Portal mobile application, which allowed participants to view personal health information, receive information tailored to identified knowledge gaps, and monitor and receive feedback on current self-management practices

Control group received usual care

Intervention included:

Continuous patient data collection includin self-assessment of asthma control and assessment of patient knowledge regarding asthma control

Individualised alerts, coaching and educational materials

Intervention group included access to "AsthmaVillage," an online community for patients with asthma

Control group did not have access to the online community, but utilised the "AsthmaDiary," an online diary for recording ICS preventer use

Intervention group utilised an application that included:

An asthma monitoring application and peak flow meter

Questionnaires and daily patient symptom score

Daily alerts and action plans based on asthma control status

The control group did not use the application

"Living Well with Asthma" website designed to

assess current level of asthma control, support

optimal medication management, challenge

attitudes and concerns around medication, and prompt use of a personal action plan

Control group received usual care

Tailored feedback and reminders based on answers to questions (at least once per month) related to asthma symptoms, medications, provider care and the asthma management plan

Control group received similar questions and feedback, but focused on preventive services unrelated to asthma control le.g. cancer screeningl
Significant improvement in self-reported 7-day Increased QoL ( $\mathrm{p}=0.037)$ and perception of adherence ( $p=0.011)$ in the intervention group versus the control group self-efficacy $(p=0.016)$ in the group compared with the control group

Not measured

No significant between-group effects on asthma-related QoL

No significant effect on asthma control

Not measured

Statistically significant improvement in ACT scores and $\mathrm{FEV}_{1}$ (in subset of patients with available before-and-after spirometry datal

Nonsignificant decrease in total number of systemic corticosteroids prescribed

No difference in self-reported medication adherence in the intervention group versus contro

Adherence improved in the intervention group $p=0.017)$ but not in the control group $(p=0.674)$

No significant between-group difference in the percentage of recommended ICS doses self-reportedly taken, nor in the equivalent beclometasone doses prescribed

No differences were observed in medication adherence between the intervention group and the control group

Not measured

Lung function parameters did not significantly differ between visits or between the intervention and control groups at each visit

No significant difference in $A C Q$ scores and mini-AQLQ scores

Significant improvement in PAM scores in the intervention group compared with the control group

Greater mean improvement in ACT score in the intervention group compared to the control group (2.3 versus $1.2, p=0.02$ )

No differences in asthma-related healthcare utilisation 


\begin{tabular}{|c|c|c|c|}
\hline Study description & Features & Effect on adherence & Other outcomes \\
\hline $\begin{array}{l}\text { PINNOCK et al. } 2013[34] \\
12 \text {-month RCT } \\
256 \text { adults with COPD }\end{array}$ & $\begin{array}{l}\text { Intervention group recorded symptoms and } \\
\text { medication use and monitored oxygen saturation } \\
\text { daily } \\
\text { Intervention included algorithm-generated alerts to } \\
\text { the clinical team based on patient input } \\
\text { Control group utilised standard self-monitoring }\end{array}$ & Not measured & $\begin{array}{l}\text { Number and mean duration of hospital } \\
\text { admissions for COPD did not differ } \\
\text { significantly between groups } \\
\text { No significant effect on HRQoL between groups }\end{array}$ \\
\hline $\begin{array}{l}\text { FARMER et al. } 2017[35] \\
12 \text {-month RCT } \\
166 \text { patients (aged } \geqslant 40 \text { years) } \\
\quad \text { with COPD }\end{array}$ & $\begin{array}{l}\text { Intervention included a daily symptom diary } \\
\text { including medication use, a Bluetooth-enabled } \\
\text { pulse oximeter with finger probe, monthly mood } \\
\text { screening questionnaires and tailored videos and } \\
\text { education based on patient entries } \\
\text { Control group received usual care }\end{array}$ & $\begin{array}{l}\text { No difference in self-reported medication } \\
\text { adherence on MARS }\end{array}$ & $\begin{array}{l}\text { No significant difference in the number of } \\
\text { exacerbations, relative risk of hospital } \\
\text { admission, QoL, self-reported smoking } \\
\text { cessation, depression, or anxiety } \\
\text { Better overall health status (measured with the } \\
\text { five level EuroQol 5-Dimension Questionnaire) } \\
\text { in the intervention group ( } p=0.03 \text { ) }\end{array}$ \\
\hline \multicolumn{4}{|c|}{$\begin{array}{l}\text { RCT: randomised controlled trial; FEV1: forced expiratory volume in } 1 \mathrm{~s} \text {; IRF: inhaler reminders and feedback; SMS: short message service; QoL: quality of life; SABA: short-acting } \\
\text { B-agonist; FeNO: exhaled nitric oxide fraction; ACT: asthma control test; ACQ: asthma control questionnaire; AQLQ: asthma quality of life questionnaire; ED: emergency department; } \\
\text { PEF: peak expiratory flow; ICS: inhaled corticosteroid; MMAS: Morisky medication adherence scale; PEFR: peak expiratory flow rate; HCP: healthcare provider; PAM: patient activation } \\
\text { measure; HRQoL: health-related quality of life; MARS: medication adherence report scale. }\end{array}$} \\
\hline
\end{tabular}


circumstances. In addition, the incorporation of information on other aspects important to patients' health, such as environmental exposures and physical activity, can further support optimal patient self-management.

\section{Penetration}

Many people in remote or low-income settings are not well supported by conventional healthcare $[47,48]$. These individuals often have the poorest disease understanding, low use of maintenance medications and the worst health outcomes [49]. The reach of wireless networks coupled with the relative reduction in cost and increased capability of mobile communications has led to its greater penetration into traditionally difficult-to-reach communities than robust conventional healthcare [47]. For example, individuals with CF living in rural or remote areas may face challenges accessing specialist care (i.e. long-travel time) [50, 51]. However, for patients with CF in remote areas of Western Australia, offering tele-health clinics via videoconferencing increased clinic visits and increased detection and treatment of exacerbations [51]. Although little research has been done to date regarding the use of digital technologies to support respiratory medicine in these settings, smartphones are increasingly being used to collect data in research studies [52-54]. A recent report highlighted the speed of deployment and value of tele-monitoring in intensive care units in Syria, indicating the potential for technologies in this sphere [55]. Another area where digital technologies could increase the penetration of healthcare expertise is in community pharmacies, where instruction from pharmacists supported by digitally enabled inhalers could establish habitual and correct inhaler use in people starting medication [56].
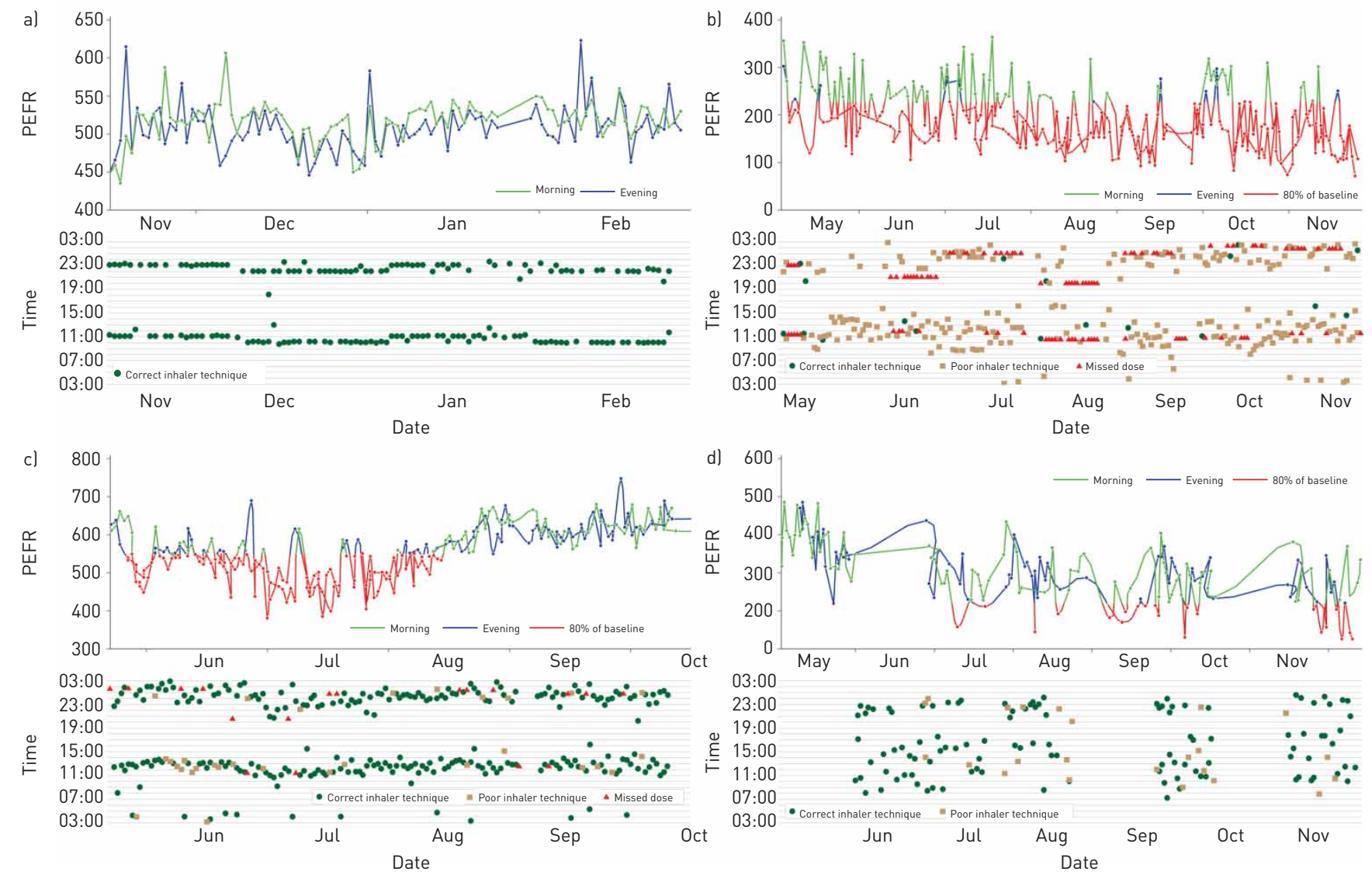

FIGURE 1 Four patterns of digitally monitored lung function, adherence and inhaler technique, as assessed by a digital audio recording device attached to the inhaler. a) A patient with well-controlled asthma with stable peak expiratory flow (PEF) and regular use of a twice daily preventer inhaler. Normal lung function (peak expiratory flow rate (PEFR)) is maintained by proficiently and regularly taken treatment (green dots on the lower graph in each panel indicate correct inhaler technique). b) A patient with poor lung function (PEFR recordings in red indicate lung function at $80 \%$ of baseline) due to poor inhaler technique (shown by orange squares) and missed doses (shown as red triangles). c) A patient with initial poor lung function who subsequently improves. Improved lung function is associated with regular and correct inhaler use. d) A patient with periods of intermittent inhaler use and poor lung function, followed by periods of improved adherence and improved lung function. Drops in lung function are associated with intermittant inhaler use and appear to prompt the patient to restart use. The absence of dots on the time graph indicates that no doses were taken. 


\section{Prediction}

A major strategy for improving health outcomes in respiratory patients is through decreasing the likelihood and magnitude of clinical deterioration, exacerbations and disease complications. Digitally collected patient data on physiology, patterns of medication adherence, trends in reliever usage and exposure to environmental pollution may identify "digital biomarkers" that help predict future exacerbations [41, 45, 57]. The use of newer data mining and analysis techniques leveraging growing mobile computing power holds particular promise to extract useful information from large datasets, as does the use of existing large and comprehensive databases as "training sets" for the development of prediction tools $[58,59]$. Thus, as technology advances, data analysis and risk stratification tools could be incorporated into smart inhalers that suggest measures to prevent severe exacerbations before they occur $[44,45]$. A recent study monitoring lung function in patients with idiopathic pulmonary fibrosis showed the value of serial measures of lung function as a way to identify different disease trajectories [60]. Additionally, in many countries, patients with respiratory conditions can also receive daily air quality alert emails or text messages intended to help them manage triggers by carrying inhalers and avoiding high pollution areas. However, adherence to behavioural recommendations following air quality alerts is poor and is an area where additional research is needed [61].

\section{Personalisation}

As described above, digital technologies are being used to gather physiological parameters over time that can help define the subtype of airway or parenchymal disease that is present $[44,62]$. They also provide unique objective insights into the behavioural aspects of a patient's life, such as his or her activity, environmental exposures and medication adherence. The latter feature may be the most important value of these technologies, particularly if patient-related factors influencing adherence are effectively matched to digital interventions that are tailored to address such determinants. Many factors impact adherence in patients with respiratory disease, highlighting the need for different approaches to address nonadherence depending on the root(s) of the issue. For example, electronic reminders and digital versions of COPD or asthma action plans could benefit patients with unintentional nonadherence due to forgetfulness or difficulty managing complex treatment regimens [42]. Forming stronger habits has been linked to better adherence in patients with CF, suggesting that strategies linking medication use to daily habits could be beneficial in patients with respiratory diseases [63]. Interventions that include a disease education component have the potential to improve adherence, which may in turn lead an individual to engage in other beneficial health behaviours. Digital technologies that collect patient information and provide insights into the specific barriers that drive patients' self-management behaviours can lead to meaningful communication between patients and HCPs, based on objective information [64]. Some factors cannot be changed or are difficult to modify (i.e. age, sex and socioeconomic status); however, barriers specific to each population can be identified and patient care tailored accordingly $[65,66]$.

To be successful, digital interventions targeting patient adherence should incorporate behavioural theory and focus on modifiable determinants relevant for the specific behaviours targeted [65]. Program development tools, such as the Intervention Mapping protocol, the Capability, Opportunity, Motivation, and Behaviour (COM-B) model, or the Theoretical Domains Framework (TDF), as well as measurement tools such as the Beliefs about Medicines Questionnaire (BMQ) and the Brief Illness Perception Questionnaire (Brief IPQ), can help match interventions to patient determinants [67, 68]. Recently, factors influencing nebuliser use in patients with CF were identified using the TDF and were used to inform development of the CFHealthHub, a digital behavioural intervention tool currently being evaluated in a large randomised trial $[69,70]$.

Furthermore, profiling tools that assign patients to an appropriate attitudinal cluster are promising approaches for grouping patients based on their beliefs and attitudes [71]. Personalising interventions to identify and target specific patient behaviours requires a baseline assessment of patient beliefs (i.e. using the BMQ or the Brief IPQ), which can then be automatically processed to ensure that the choice of patient messages, channels of communication and digital interventions are matched to the specific drivers of suboptimal adherence that are relevant to the patient [17].

In addition to timing of medication intake, optimal adherence to inhaled medications requires effective inhaler technique to ensure appropriate medication deposition in the lungs [72]. The estimated prevalence of poor inhaler technique ranges from $14 \%$ to $90 \%$ depending on the device and context [73], and is caused by a lack of knowledge on how to use the inhaler, lack of motivation to learn or implement proper technique and the need for repeated training. The use of multiple types of inhaler devices is associated with a higher prevalence of errors. Many inhaler errors, including general errors (i.e. not exhaling, not holding breath and inappropriate inhalation speed) and device-specific errors (i.e. dose preparation for dry powder inhalers and coordination problems for metered dose inhalers), are associated with reduced 
asthma symptom control and increased rates of exacerbations [74, 75]. Despite efforts invested in education, training and device development, little progress has been made to improve inhaler use over the past 40 years [76]. Recently, devices have been developed that can detect inadequate inhaler technique, helping to identify patients requiring additional training $[1,77,78]$. Some inhalers can also provide feedback directly to patients, helping them optimise their technique and, when used in newly diagnosed patients, could help them to develop good habits from the outset. When lack of motivation is at the root of a patient's poor inhaler use, combining digital monitoring and feedback on inhaler technique with other strategies (e.g. education on the importance of proper medication uptake) could prove beneficial.

Other critical factors for a successful tailored approach will involve delivering digital patient-related information to a patient's clinical team to allow for meaningful conversations during medical visits. Ensuring that tailoring is dynamic may also be important since factors that influence patients' adherence can change over time. For example, a patient's initial beliefs about treatment may change after experiencing side effects and effective digital interventions should have the capacity to detect and respond to these changes over time in a flexible way (see figures $1 \mathrm{c}$ and $1 \mathrm{~d}$ ).

The flexible nature of digital technologies provides an opportunity to develop generalisable core features and customisable elements to meet the individual patient's needs, responding to both patient preferences and the clinician's requirements. The aim would be to create interactive and adaptable digital versions of individual treatment action plans that use personal rather than population thresholds for changes in treatment, examples of which are already available [35]. Key features such as educational content could be customised to facilitate engagement based on a patient's health literacy, treatment perceptions and disease experience.

\section{Key considerations toward successful implementation and uptake of digital applications}

Digital technologies have the ability to improve the precision, penetration, prediction and personalisation of respiratory care. However, for this potential to be realised, several conditions need to be in place. A strong evidence base that represents the users is required, as are strategies for how technologies will be integrated into health systems, together with the appropriate regulatory standards.

\section{How strong is the evidence base underpinning new technologies?}

Research on critical factors that could impact the use of digital technologies to manage adherence in the clinical setting has not kept pace with the rapid advances in the technologies themselves, in part because the pace at which research is funded, completed and published cannot keep up with the rapid evolution of available technologies $[79,80]$. Frameworks have been published that describe key elements to be included in studies describing and evaluating new digital applications [12, 80-83]. However, most published studies to date have not measured up to these standards, with the majority being of short duration and not describing in sufficient detail the features of the technologies or how they were evaluated $[81,82,84]$. To proceed with widespread implementation of newer technologies, we need a more complete and detailed understanding of factors related to the use of digital interventions by patients and clinicians, the impact of these interventions on HCP workload and their integration with existing services, as well as their economic impact.

\section{Considering the intended users of the interventions}

To avoid the restricted entry criteria and rigid ecology of care issues that characterise traditional trials of medications, studies of digital interventions must reflect the user base and the usual clinical environment, and not simply study early technology adopters using teaching hospitals. This is especially important since patient engagement and sustained use in the real-world setting are critical factors for the success of digital technologies. Unfortunately, high rates of discontinuation are common with digital tools [13, 52]. Therefore, research is needed to understand the reasons for initiation, persistence and discontinuation of use. Many authors have provided useful overviews of this problem along with guidelines for promoting more effective engagement $[85,86]$. Recording and monitoring events with reminders and feedback on performance are often desirable features $[87,88]$. However, the intrusive nature of this monitoring and subsequent judgment may lead some patients to disengage. For example, a study of smart inhaler use in children with asthma showed a greater rate of lost or damaged inhalers than might reasonably be expected in usual practice or in the control group [13]. Continuous improvement and customisation is also possible, starting from a basic user experience and incorporating user feedback features [17, 89-91].

\section{Integrating digital technologies into existing healthcare systems}

Huge amounts of data will be generated by new connected devices and mobile applications. For example, an asthma specialist with a moderate caseload would receive data on many thousands of actuations per 
year if all inhalers were digitally equipped. Despite offering great promise in improving clinical outcomes, handling this volume of data is somewhat daunting. At a basic level, information from connected devices should integrate with patients' electronic medical records to facilitate decision making in the same way clinicians view blood test results. Devices or mobile software that demand a stand-alone interface are less practical and will be short-lived. Progress is being achieved by improving standardisation and facilitating transfer of information between devices and different healthcare settings (by consortia such as INTEROPen; www.interopen.org), as well as by the development of secure communication formats (by systems such as blockchain; www.blockchain.com).

Integrating digital technology into usual care may also involve substantial changes in routine clinician practices. These changes need to be approached using the same behavioural principles applied to patients: clearly define the behaviours requiring change, identify relevant determinants and incorporate behaviour change techniques that effectively target these behaviours and determinants [92]. Additionally, evidence-based data from clinical trials are needed so that clinicians can be confident in the effectiveness and cost-effectiveness of proposed digital interventions. Furthermore, preparing organisations for technology-supported care of chronic conditions is likely to represent a financial burden with significant scope for costly challenges, with many institutions currently lacking the appropriate infrastructure to support digital innovation (figure 2) [93]. Additionally, the handling of large volumes of data collected from different digital devices and at different medical institutions is a necessary step to allow meaningful aggregation of information [94].

\section{Developing appropriate regulatory standards for digital technologies}

Meeting the regulatory standards for medicines is costly and time consuming, and is possible only with robust intellectual property protection to secure future income. It is clear that the situation in terms of regulatory needs for new technologies is very different than for standard medications [95]. As devices and software can be readily mimicked, there is a race to market to secure an initial income. Offerings are therefore marketed as lifestyle rather than health products and are of myriad and usually low quality [84]. As such, regulatory standards for digital technologies may need to be appropriately tailored to encourage development of medically focused digital applications.

Finally, the challenges in maintaining anonymity and data security for both data in transit and stored data are a concern across all medical specialties [96]. A full discussion of this complex topic is beyond this

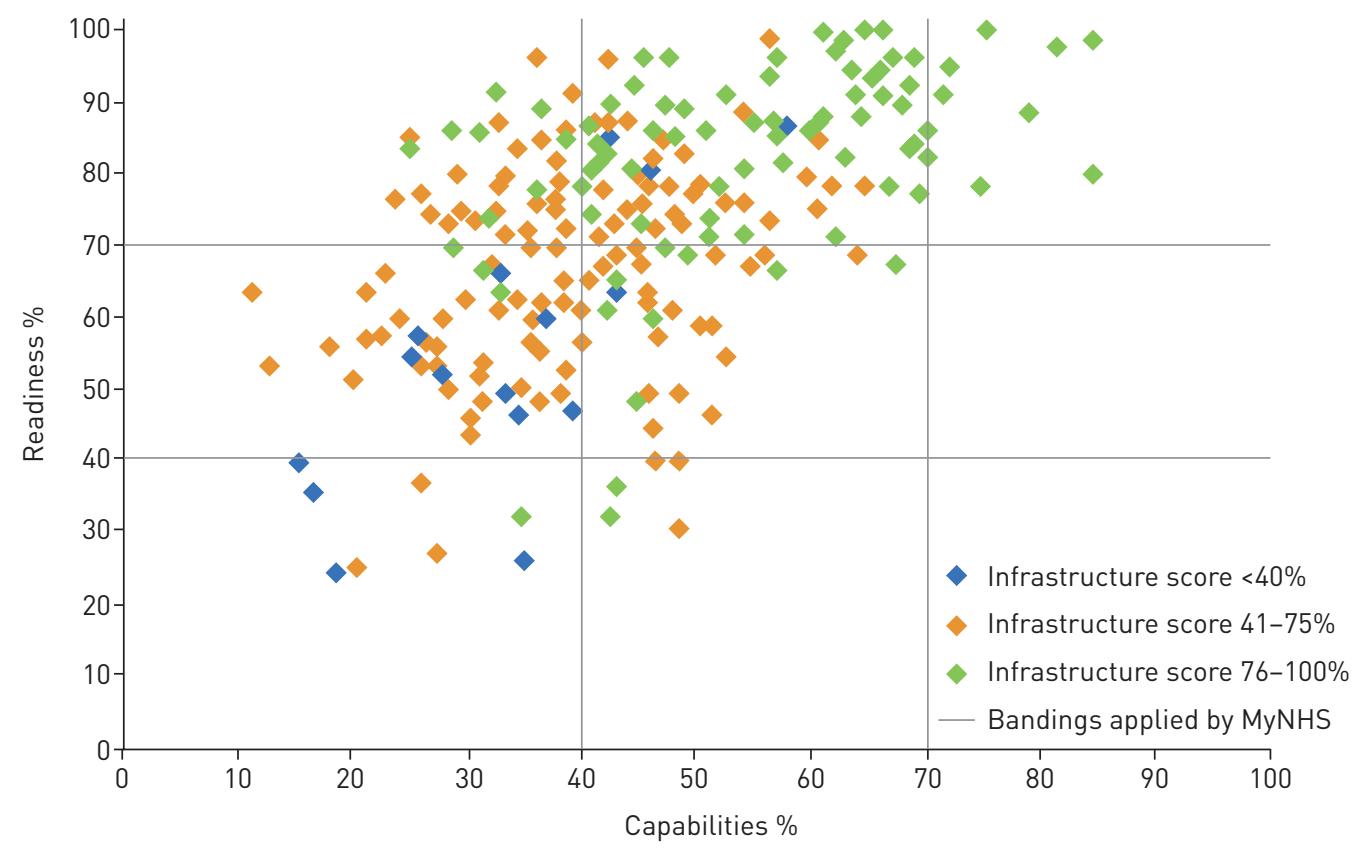

FIGURE 2 Results of the Digital Maturity Self-Assessment survey in 2016, measuring how well secondary care providers in England are making use of digital technology to achieve a health and care system that is paper-free at the point of care. Readiness indicates how well providers are able to plan and deploy digital services, while capabilities indicate whether providers have staff with the digital skills needed. The infrastructure score is based on whether providers have the right technology in place. Data was from the National Health Service (NHS), England. Reproduced with permission of the rights holder, Royal College of Physicians from [93]. 
article, but it appears we could well see a transition from large commercial institutions storing data on many patients to individuals holding their own data (e.g. the personal data management system described by Mortier et al. [97]). This scenario provides the strongest protection to end-users in terms of deciding who will use their data, but could compromise the potential for using aggregated de-identified information for research purposes.

\section{Conclusions}

For patients, clinicians and researchers, digital technologies such as those addressing adherence and inhaler technique offer the opportunity to advance personalised care for patients with respiratory diseases. Longitudinal, real-time data collected through digital platforms can provide a precise understanding of the interaction of a patient's adherence, symptoms and disease course, which can be used to develop and support adherence management strategies. Digital technologies also offer the possibility of extending the reach of healthcare into underserved communities, where adherence is often the poorest, or of empowering HCPs who are not specialists. Digital biomarkers may be developed that link adherence with physiological parameters and environmental exposures to help predict events such as loss of symptom control or exacerbations. Finally, digital interventions can be tailored to address individual patient determinants of adherence.

For such approaches to be successful, strategies to promote uptake and implementation of new technologies need to be developed in parallel. These include guideline-based design and evaluation of new technologies to develop a strong evidence base underpinning digital interventions. The features and capabilities of digital interventions should also be designed with the end user in mind to promote long-term use. Strategies to facilitate uptake by HCPs also need to be implemented to allow for their successful integration into existing healthcare systems. Finally, the appropriate regulatory processes for digital interventions remain to be identified, and appropriate tools and security measures are needed to manage the large amounts of data generated. Ongoing investigation of digital solutions will establish their place in the management of asthma and COPD.

Author contributions: All listed authors meet the criteria for authorship set forth by the International Committee for Medical Journal Editors. All authors agree to be accountable for all aspects of this review article. All authors participated in determining the content included in the review and in drafting the article and revising it critically for important intellectual content. All authors approved the final version of the article for submission.

Conflict of interest: J.D. Blakey reports personal fees (for an advisory board meeting) from Teva, personal fees (for travel and lectures) and non-financial support from Napp, personal fees (for a presentation) from Novartis, personal fees and non-financial support (for travel and lectures) from AstraZeneca, and personal fees and non-financial support (for travel and lectures) from Boehringer Ingelheim, outside the submitted work. B.G. Bender has nothing to disclose. A.L. Dima reports grants and non-financial support (for travel and research) from Respiratory Effectiveness Group, outside the submitted work. J. Weinman reports personal fees from Atlantis Healthcare, Boehringer Ingelheim, Chugai/ Roche, Ferring, Sanofi and Teva, and grants from Merck, outside the submitted work. G. Safioti is an employee of Teva. R.W. Costello has received funding for research from Aerogen and GSK, speaker's and consultancy fees from Aerogen, Boehringer Inghelheim, AstraZeneca, GSK, Novartis and Teva. He has licensed an acoustic device to assess adherence to Vitalograph and has a patent pending for the identification of inhaler use (P10961USPC) and two pending for methods to assess adherence and remotely predict exacerbations.

Support Statement: Medical editorial support (Sarah Hummasti) and graphic services were provided by AOIC LLC (Exton, PA, USA) and were funded by Teva Pharmaceutical Industries Ltd. Funding information for this article has been deposited with the Crossref Funder Registry.

\section{References}

1 Chan AH, Reddel HK, Apter A, et al. Adherence monitoring and e-health: how clinicians and researchers can use technology to promote inhaler adherence for asthma. J Allergy Clin Immunol Pract 2013; 1: 446-454.

2 Bosnic-Anticevich S, Chrystyn H, Costello RW, et al. The use of multiple respiratory inhalers requiring different inhalation techniques has an adverse effect on COPD outcomes. Int J Chron Obstruct Pulmon Dis 2017; 12: 59-71.

3 van Boven JF, Ryan D, Eakin MN, et al. Enhancing respiratory medication adherence: the role of health care professionals and cost-effectiveness considerations. J Allergy Clin Immunol Pract 2016; 4: 835-846.

4 Vrijens B, Dima AL, Van Ganse E, et al. What we mean when we talk about adherence in respiratory medicine. J Allergy Clin Immunol Pract 2016; 4: 802-812.

5 Chan AH, Stewart AW, Harrison J, et al. The effect of an electronic monitoring device with audiovisual reminder function on adherence to inhaled corticosteroids and school attendance in children with asthma: a randomised controlled trial. Lancet Respir Med 2015; 3: 210-219.

6 Charles T, Quinn D, Weatherall M, et al. An audiovisual reminder function improves adherence with inhaled corticosteroid therapy in asthma. J Allergy Clin Immunol 2007; 119: 811-816.

7 Milgrom H, Bender B, Ackerson L, et al. Noncompliance and treatment failure in children with asthma. J Allergy Clin Immunol 1996; 98: 1051-1057.

8 Morton RW, Elphick HE, Edwards E, et al. Investigating the feasibility of text message reminders to improve adherence to nebulized medication in children and adolescents with cystic fibrosis. Patient Prefer Adherence 2017; 11: 861-869. 
9 Burgess SW, Sly PD, Devadason SG. Providing feedback on adherence increases use of preventive medication by asthmatic children. J Asthma 2010; 47: 198-201.

10 Foster JM, Usherwood T, Smith L, et al. Inhaler reminders improve adherence with controller treatment in primary care patients with asthma. J Allergy Clin Immunol 2014; 134: 1260-1268.

11 Vasbinder EC, Goossens LM, Rutten-van Molken MP, et al. e-Monitoring of Asthma Therapy to Improve Compliance in children (e-MATIC): a randomised controlled trial. Eur Respir J 2016; 48: 758-767.

12 Merchant RK, Inamdar R, Quade RC. Effectiveness of population health management using the propeller health asthma platform: a randomized clinical trial. J Allergy Clin Immunol Pract 2016; 4: 455-463.

13 Morton RW, Elphick HE, Rigby AS, et al. STAAR: a randomised controlled trial of electronic adherence monitoring with reminder alarms and feedback to improve clinical outcomes for children with asthma. Thorax 2017; 72: 347-354.

14 Strandbygaard U, Thomsen SF, Backer V. A daily SMS reminder increases adherence to asthma treatment: a three-month follow-up study. Respir Med 2010; 104: 166-171.

15 Prabhakaran L, Chee WY, Chua KC, et al. The use of text messaging to improve asthma control: a pilot study using the mobile phone short messaging service (SMS). J Telemed Telecare 2010; 16: 286-290.

16 Lv Y, Zhao H, Liang Z, et al. A mobile phone short message service improves perceived control of asthma: a randomized controlled trial. Telemed J E Health 2012; 18: 420-426.

17 Petrie KJ, Perry K, Broadbent E, et al. A text message programme designed to modify patients' illness and treatment beliefs improves self-reported adherence to asthma preventer medication. Br J Health Psychol 2012; 17: 74-84.

18 Kolmodin MacDonell K, Naar S, Gibson-Scipio W, et al. The Detroit Young Adult Asthma Project: pilot of a technology-based medication adherence intervention for African-American emerging adults. $J$ Adolesc Health 2016; 59: 465-471

19 Britto MT, Rohan JM, Dodds CM, et al. A randomized trial of user-controlled text messaging to improve asthma outcomes. Clin Pediatr (Phila) 2017; 56: 1336-1344.

20 Akrom Nurwijayanti A. Brief counseling and mobile phone short message service (SMS) increase patient compliance. Int J Pharm Med Biol Sc 2015; 4: 175-179.

21 van der Meer V, Bakker MJ, van den Hout WB, et al. Internet-based self-management plus education compared with usual care in asthma: a randomized trial. Ann Intern Med 2009; 151: 110-120.

22 Liu WT, Huang $\mathrm{CD}$, Wang $\mathrm{CH}$, et al. A mobile telephone-based interactive self-care system improves asthma control. Eur Respir J 2011; 37: 310-317.

23 Ryan D, Price D, Musgrave SD, et al. Clinical and cost effectiveness of mobile phone supported self monitoring of asthma: multicentre randomised controlled trial. BMJ 2012; 344: e1756.

24 Farooqui N, Phillips G, Barrett C, et al. Acceptability of an interactive asthma management mobile health application for children and adolescents. Ann Allergy Asthma Immunol 2015; 114: 527-529.

25 Mosnaim G, Li H, Martin M, et al. A tailored mobile health intervention to improve adherence and asthma control in minority adolescents. J Allergy Clin Immunol Pract 2015; 3: 288-290.

26 Cingi C, Yorgancioglu A, Cingi CC, et al. The "physician on call patient engagement trial" (POPET): measuring the impact of a mobile patient engagement application on health outcomes and quality of life in allergic rhinitis and asthma patients. Int Forum Allergy Rhinol 2015; 5: 487-497.

27 Johnson KB, Patterson BL, Ho YX, et al. The feasibility of text reminders to improve medication adherence in adolescents with asthma. J Am Med Inform Assoc 2016; 23: 449-455.

28 Ahmed S, Ernst P, Bartlett SJ, et al. The effectiveness of web-based asthma self-management system, My Asthma Portal (MAP): a pilot randomized controlled trial. J Med Internet Res 2016; 18: e313.

29 Cook KA, Modena BD, Simon RA. Improvement in asthma control using a minimally burdensome and proactive smartphone application. J Allergy Clin Immunol Pract 2016; 4: 730-737 e731.

30 Koufopoulos JT, Conner MT, Gardner PH, et al. A web-based and mobile health social support intervention to promote adherence to inhaled asthma medications: randomized controlled trial. J Med Internet Res 2016; 18: e122.

31 Kim MY, Lee SY, Jo EJ, et al. Feasibility of a smartphone application based action plan and monitoring in asthma. Asia Pac Allergy 2016; 6: 174-180.

32 Morrison D, Wyke S, Saunderson K, et al. Findings from a pilot randomised trial of an asthma internet self-management intervention (RAISIN). BMJ Open 2016; 6: e009254.

33 Pool AC, Kraschnewski JL, Poger JM, et al. Impact of online patient reminders to improve asthma care: a randomized controlled trial. PLoS One 2017; 12: e0170447.

34 Pinnock H, Hanley J, McCloughan L, et al. Effectiveness of telemonitoring integrated into existing clinical services on hospital admission for exacerbation of chronic obstructive pulmonary disease: researcher blind, multicentre, randomised controlled trial. BMJ 2013; 347: f6070.

35 Farmer A, Williams V, Velardo C, et al. Self-management support using a digital health system compared with usual care for chronic obstructive pulmonary disease: randomized controlled trial. J Med Internet Res 2017; 19: e144.

36 Geller DE, Madge S. Technological and behavioral strategies to reduce treatment burden and improve adherence to inhaled antibiotics in cystic fibrosis. Respir Med 2011; 105: Suppl. 2, S24-S31.

37 Seheult JN, O'Connell P, Tee KC, et al. The acoustic features of inhalation can be used to quantify aerosol delivery from a Diskus dry powder inhaler. Pharm Res 2014; 31: 2735-2747.

38 Sulaiman I, Cushen B, Greene G, et al. Objective assessment of adherence to inhalers by patients with chronic obstructive pulmonary disease. Am J Respir Crit Care Med 2017; 195: 1333-1343.

39 Kikidis D, Konstantinos V, Tzovaras D, et al. The digital asthma patient: the history and future of inhaler based health monitoring devices. J Aerosol Med Pulm Drug Deliv 2016; 29: 219-232.

40 Lucas RW, Dees J, Reynolds R, et al. Cloud-computing and smartphones: tools for improving asthma management and understanding environmental triggers. Ann Allergy Asthma Immunol 2015; 114: 431-432.

41 Shah SA, Velardo C, Farmer A, et al. Exacerbations in chronic obstructive pulmonary disease: identification and prediction using a digital health system. J Med Internet Res 2017; 19: e69.

42 van Boven JF, Trappenburg JC, van der Molen T, et al. Towards tailored and targeted adherence assessment to optimise asthma management. NPJ Prim Care Respir Med 2015; 25: 15046. 
$43 \mathrm{Hu}$ Y, Kim EG, Cao G, et al. Physiological acoustic sensing based on accelerometers: a survey for mobile healthcare. Ann Biomed Eng 2014; 42: 2264-2277.

44 Honkoop PJ, Simpson A, Bonini M, et al. MyAirCoach: the use of home-monitoring and mHealth systems to predict deterioration in asthma control and the occurrence of asthma exacerbations; study protocol of an observational study. BMJ Open 2017; 7: e013935.

45 Killane I, Sulaiman I, MacHale E, et al. Predicting asthma exacerbations employing remotely monitored adherence. Healthc Technol Lett 2016; 3: 51-55.

46 Lindsay JT, Heaney LG. Non-adherence in difficult asthma and advances in detection. Expert Rev Respir Med 2013; 7: 607-614.

47 Graham GN, Ostrowski M, Sabina AB. Population health-based approaches to utilizing digital technology: a strategy for equity. J Public Health Policy 2016; 37: Suppl. 2, 154-166.

48 Valet RS, Perry TT, Hartert TV. Rural health disparities in asthma care and outcomes. J Allergy Clin Immunol 2009; 123: 1220-1225.

49 Himes BE, Weitzman ER. Innovations in health information technologies for chronic pulmonary diseases. Respir Res 2016; 17: 38.

50 Roberts JM, Wilcox PG, Quon BS. Evaluating adult cystic fibrosis care in BC: disparities in access to a multidisciplinary treatment centre. Can Respir J 2016; 2016: 8901756.

51 Wood J, Mulrennan S, Hill K, et al. Telehealth clinics increase access to care for adults with cystic fibrosis living in rural and remote Western Australia. J Telemed Telecare 2017; 23: 673-679.

52 Chan YY, Wang P, Rogers L, et al. The Asthma Mobile Health Study, a large-scale clinical observational study using ResearchKit. Nat Biotechnol 2017; 35: 354-362.

53 McConnell MV, Shcherbina A, Pavlovic A, et al. Feasibility of obtaining measures of lifestyle from a smartphone app: the MyHeart Counts Cardiovascular Health Study. JAMA Cardiol 2017; 2: 67-76.

54 Ritter S. Apple's research kit development framework for Iphone apps enables innovative approaches to medical research data collection. J Clin Trials 2015; 5: e120.

55 Moughrabieh A, Weinert C. Rapid deployment of international tele-intensive care unit services in war-torn Syria. Ann Am Thorac Soc 2016; 13: 165-172.

56 Giraud V, Allaert FA, Roche N. Inhaler technique and asthma: feasability and acceptability of training by pharmacists. Respir Med 2011; 105: 1815-1822.

57 Jenkins CR, Postma DS, Anzueto AR, et al. Reliever salbutamol use as a measure of exacerbation risk in chronic obstructive pulmonary disease. BMC Pulm Med 2015; 15: 97.

58 Blakey JD, Price DB, Pizzichini E, et al. Identifying risk of future asthma attacks using UK medical record data: a Respiratory Effectiveness Group initiative. J Allergy Clin Immunol Pract 2017: 5;1015-1024.

59 Reymann MP, Dorschky E, Groh BH, et al. Blood glucose level prediction based on support vector regression using mobile platforms. Conf Proc IEEE Eng Med Biol Soc 2016; 2016: 2990-2993.

60 Russell AM, Adamali H, Molyneaux PL, et al. Daily home spirometry: an effective tool for detecting progression in idiopathic pulmonary fibrosis. Am J Respir Crit Care Med 2016; 194: 989-997.

61 D'Antoni D, Smith L, Auyeung V, et al. Psychosocial and demographic predictors of adherence and non-adherence to health advice accompanying air quality warning systems: a systematic review. Environ Health 2017; 16: 100 .

62 Bafadhel M, Pavord ID, Russell REK. Eosinophils in COPD: just another biomarker? Lancet Respir Med 2017; 5 747-759.

63 Hoo ZH, Gardner B, Arden MA, et al. Role of habit in treatment adherence among adults with cystic fibrosis. Thorax 2018; in press [https://doi.org/10.1136/thoraxjnl-2017-211453].

64 Blackstock FC, ZuWallack R, Nici L, et al. Why don't our patients with chronic obstructive pulmonary disease listen to us? The enigma of Nonadherence. Ann Am Thorac Soc 2016; 13: 317-323.

65 Dima AL, de Bruin M, Van Ganse E, et al. Mapping the asthma care process: implications for research and practice. J Allergy Clin Immunol Pract 2016; 4: 868-876.

66 Lakhanpaul M, Bird D, Culley L, et al. The use of a collaborative structured methodology for the development of a multifaceted intervention programme for the management of asthma (the MIA project), tailored to the needs of children and families of South Asian origin: a community-based, participatory study. Health Serv Deliv Res 2014; 2 (28). www.journalslibrary.nihr.ac.uk/hsdr/hsdr02280/\#/full-report

67 Allemann SS, Nieuwlaat R, van den Bemt BJ, et al. Matching adherence interventions to patient determinants using the theoretical domains framework. Front Pharmacol 2016; 7: 429.

68 Jackson C EL, Barber N, Weinman J. Applying COM-B to medication adherence: a suggested framework for research and interventions. Eur Heal Psychol 2014; 16: 7-17.

69 CFHealthHub. Development and evaluation of an intervention to support adherence to treatment in adults with cystic fibrosis. www.sheffield.ac.uk/scharr/sections/dts/ctru/cfhealthhub Date last accessed: July 18, 2018.

70 Arden MA, Drabble SJ, O'cathain A, et al. WS 16.1 ACtiF study: understanding adherence to nebuliser treatment in adults with cystic fibrosis using the theoretical domains framework. J Cyst Fibros 2016; 15: Suppl. 1, S26.

71 David-Wang A, Price D, Cho SH, et al. Development and validation of an attitudinal-profiling tool for patients with asthma. Allergy Asthma Immunol Res 2017; 9: 43-51.

72 Sulaiman I, Seheult J, Sadasivuni N, et al. The impact of common inhaler errors on drug delivery: investigating critical errors with a dry powder inhaler. I Aerosol Med Pulm Drug Deliv 2017; 30: 247-255.

73 Braido F, Chrystyn H, Baiardini I, et al. "Trying, but failing" - the role of inhaler technique and mode of delivery in respiratory medication adherence. J Allergy Clin Immunol Pract 2016; 4: 823-832.

74 Melani AS, Bonavia M, Cilenti V, et al. Inhaler mishandling remains common in real life and is associated with reduced disease control. Respir Med 2011; 105: 930-938.

75 Price DB, Roman-Rodriguez M, McQueen RB, et al. Inhaler errors in the CRITIKAL study: type, frequency, and association with asthma outcomes. J Allergy Clin Immunol Pract 2017; 5: 1071-1081.

76 Sanchis J, Gich I, Pedersen S, et al. Systematic review of errors in inhaler use: has patient technique improved over time? Chest 2016; 150: 394-406.

77 D'Arcy S, MacHale E, Seheult J, et al. A method to assess adherence in inhaler use through analysis of acoustic recordings of inhaler events. PLoS One 2014; 9: e98701. 
Sulaiman I, Seheult J, MacHale E, et al. Irregular and ineffective: a quantitative observational study of the time and technique of inhaler use. J Allergy Clin Immunol Pract 2016; 4: 900-909.

79 Bender BG. Technology interventions for nonadherence: new approaches to an old problem. J Allergy Clin Immunol Pract 2018; 6: 794-800.

80 Murray E, Hekler EB, Andersson G, et al. Evaluating digital health interventions: key questions and approaches. Am J Prev Med 2016; 51: 843-851.

81 Agarwal S, LeFevre AE, Lee J, et al. Guidelines for reporting of health interventions using mobile phones: mobile health (mHealth) evidence reporting and assessment (mERA) checklist. BMJ 2016; 352: i1174.

82 Eysenbach G, Group C-E. CONSORT-EHEALTH: improving and standardizing evaluation reports of web-based and mobile health interventions. J Med Internet Res 2011; 13: e126.

83 Wilhide IC, Peeples MM, Anthony Kouyate RC. Evidence-based mHealth chronic disease mobile app intervention design: development of a framework. JMIR Res Protoc 2016; 5: e25.

84 Huckvale K, Morrison C, Ouyang J, et al. The evolution of mobile apps for asthma: an updated systematic assessment of content and tools. BMC Med 2015; 13: 58.

85 Perski O, Blandford A, West R, et al. Conceptualising engagement with digital behaviour change interventions: a systematic review using principles from critical interpretive synthesis. Transl Behav Med 2017; 7: 254-267.

86 Yardley L, Spring BJ, Riper $\mathrm{H}$, et al. Understanding and promoting effective engagement with digital behavior change interventions. Am J Prev Med 2016; 51: 833-842.

87 Brouwer W, Kroeze W, Crutzen R, et al. Which intervention characteristics are related to more exposure to internet-delivered healthy lifestyle promotion interventions? A systematic review. J Med Internet Res 2011; 13 : e2.

88 Checchi KD, Huybrechts KF, Avorn J, et al. Electronic medication packaging devices and medication adherence: a systematic review. JAMA 2014; 312: 1237-1247.

89 DeWalt DA, Malone RM, Bryant ME, et al. A heart failure self-management program for patients of all literacy levels: a randomized, controlled trial [ISRCTN11535170]. BMC Health Serv Res 2006; 6: 30.

90 Velardo C, Shah SA, Gibson O, et al. Digital health system for personalised COPD long-term management. BMC Med Inform Decis Mak 2017; 17: 19.

91 Yin HS, Gupta R, Mendelsohn AL, et al. Use of a low literacy written action plan to improve parent understanding of pediatric asthma management: a randomized controlled study. J Asthma 2017; 54: 919-929.

92 Johnson MJ, May CR. Promoting professional behaviour change in healthcare: what interventions work, and why? A theory-led overview of systematic reviews. BMJ Open 2015; 5: e008592.

93 Johnston DS. Digital maturity: are we ready to use technology in the NHS. Future Healthcare Journal 2017; 4: 189-192.

94 Dimitrov DV. Medical internet of things and big data in healthcare. Healthc Inform Res 2016; 22: 156-163.

95 Gold ER, Kaplan W, Orbinski J, et al. Are patents impeding medical care and innovation? PLoS Med 2010; 7: e1000208.

96 Taitsman JK, Grimm CM, Agrawal S. Protecting patient privacy and data security. N Engl J Med 2013; 368: 977-979.

97 Mortier R, Zhao J, Crowcroft J, et al. Personal data management with the Databox: what's inside the box? Proceedings of the 2016 ACM Workshop on Cloud-Assisted Networking 2016; 49-54. https://dl.acm.org/citation. cfm?id $=3010082$ 